\title{
Unearthing principal parameters to ameliorate score in National Institutional Ranking Framework deploying Deep Learning approach
}

\author{
M.Pradeep ${ }^{1}$, Terrance Frederick Fernandez ${ }^{2}$, K. ShanthaKumari ${ }^{3}$, \\ A. Jerold ${ }^{4}$, M. Rajesh Kumar ${ }^{5}$ \\ ${ }^{1}$ Assistant Professor, Department of Information Technology, Rajiv Gandhi College of Engineering and \\ Technology, Puducherry \\ ${ }^{2}$ Associate Professor, Department of Computer Science \& Engineering, Dhanalakshmi Srinivasan College \\ of Engineering \& Technology \\ ${ }^{3}$ Associate Professor, Department of Computer Science \& Engineering, Rajiv Gandhi College of Engineering and \\ Technology, Puducherry \\ ${ }^{4}$ Student, Department of Information Technology, Rajiv Gandhi College of Engineering and Technology, \\ Puducherry \\ ${ }^{5} \mathrm{PhD}$ Scholar, Department of Radiodiagnosis, SBVU, Puducherry
}

\begin{abstract}
The National Institutional Ranking Framework (NIRF) is a ranking framework followed nationwide and adopted by the Educational Ministry, Government of India, for ranking Higher Education Institutions (HEIs) and envision students to better choose their higher educational arena. This nationalized Framework was ratified and launched by the Minister of Human Resource Development. Since 2016, NIRF publishes 100 HEIs in ranking order based on multiple parameters like teaching, learning, research and many more.In this paper, we identify crucial parameters which influence the overall score of an institution. Our proposed deep learning architecture which employs Neural Network predicts with the improvement of what parametric value, an HEI could rise up the ladder in the ranking framework. Furthermore, we implement our work using Orange data science toolkit followed by python framework and elaborately discuss the results.
\end{abstract}

Keywords: Deep Learning, National Institutional Ranking Framework, Neural Networks

\section{$1 \quad$.INTRODUCTION}

India, a country famed for its rich culture and heritage is now popular for many other reasons. Backed with over 356 million youths, the second most populous sub-continent has highest number of universities. Yes, it has 4,381 universities succeeded by United States with 3,254 HEIs [1]. On 29th September 2015, Ministry of Human Resource Development under the Government of India launched National Institutional Ranking Framework (NIRF) [1]. One significant aspect of NIRF against all other ranking frameworks is the openness of data upon which the ranking is made. NIRF is just one among many ranking strategies in our country [5]. To name few, we do have NBA, NAAC, ARIIA etc. Every ranking system is usually backed-up with multiple ranking parameters. There are 5 ranking parameters that govern the overall NIRF score of an HEI. They are

- $\quad$ Teaching, Learning \& Resources (TLR)

- $\quad$ Research and Professional Practices (RP)

- $\quad$ Graduation Outcomes (GO)

- $\quad$ Outreach and Inclusivity (OI)

- $\quad$ Perception (PR)

The ranking parameters are uniform across all ranking categories in NIRF. There are 9 categories (disciplines) in NIRF like Engineering, management, medical, architecture, law and many more. There is also an overall category in which all disciplines are collectively ranked [9]. 


\section{DOI: $10.17148 /$ IARJSET.2021.8841}

Around 1400 Indian institutions applied for NIRF in the year 2016. By the next year, 3000 HEIs applied for the same [6]. Last year, in 2020, around 4000 institutions competed in the ranking, which was $20 \%$ higher than the scenario in 2019 [8]. Up recently, we can browse through last year's ranked lists at the website and for the first time, the Dental institutes were placed under an individual ranking ledger [6].

The prime objective of the manuscript is to create a business model employing Deep learning algorithm to leverage better rank in NIRF. Deep learning algorithms were extensively used to predict outcomes in varied scenarios [5-6].

\section{RELATED WORKS}

This section of the manuscript ventilates the works carried out by the researchers in relation to NIRF and ranking of educational Institutions in general.The works mentioned in [5, 6 and 7] were engineered solely towards Government of India's NIRF. An immaculate assessment of the ranking parameters and scoring methodologies (which were debriefed in SECTION 1) used in NIRF was done in [1] and the authors were keen to spread awareness about the ranking framework to the academia through this work.

Later in the year 2017, A. M. and M. Nagabhushanam et.al.., architected a policy recommendation to the MHRD, Government of India, upon existing NIRF. The reason behind their curtain was to improve the quality of Indian education among global Institutes [2]. The motivation came from a study from [8] which made a disclosure that less than 10 HEIs have made it among the top 100 universities worldwide. The Ideal and the Minimum Attainment Scores in each ranking parameter for NIRF was disclosed in [7] that could aid HEIs to apply for the forthcoming competition. They took up a specific case study of Thailand university thereby sorting out relationship between number of back-links, page view average, visit durations times, percentage of bounce rate and impact ranking of webometrics. Secondly, down in Indonesia, B. S. Rintyarna and his team was keen on ranking the technology readiness level of universities in Indonesia [6]. Their work was successful and resulted in $91.6 \%$ of similarity with their national ranking scheme.

\section{3 .OUR PROPOSED METHODOLOGY}

The previous section presented related works pertaining to NIRF grading of HEIs or ranking of institutions in general. It is inferred that none of the works were concerned with improving of the ranking scores for the institutions. This was our motivation to develop a deep learning based business model to enable HEIs to enrich their ranking parameter in the forthcoming academic year. In this section, we will debrief on how institutions can improve their NIRF scores using our methodology.

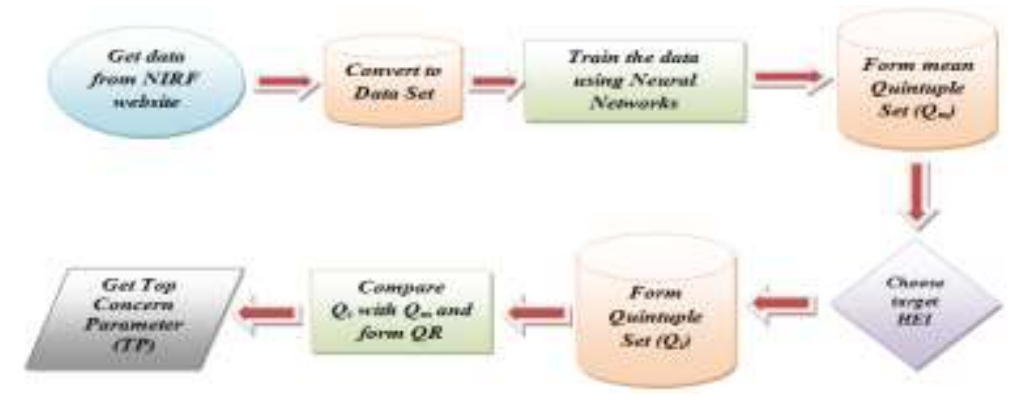

Fig.1. Our Proposed Architecture

At the first section of the paper, we have mentioned about the five principal ranking parameters in NIRF. These parameters are further subdivided into 17 sub-parameters, which serve as the fulcrum input for our work. The ranking parametric ledger of NIRF is furnished in their official site and is referred from [5]. NIRF website not only publishes the ranking order list with the overall scores but also the individual ranking parametric scores.

The entire 17 value set for each college is fetched and are converted to Data sets to train the data. Say for instance, in the year 2020, there were 40 rankings for medical institutions across the country and for this scenario, we take (40x17) values and convert them into data sets. A data set for each HEI contains a quintuple [15]. A quintuple contains five values, one for each ranking parameter. Let us consider Quintuple $\left(\mathrm{Q}_{\mathrm{i}}\right)=\left(\mathrm{t}_{\mathrm{i}}, \mathrm{r}_{\mathrm{i}}, \mathrm{g}_{\mathrm{i}}, \mathrm{O}_{\mathrm{i}}, \mathrm{p}_{\mathrm{i}}\right)$ and $\mathrm{i}$ can take values from 1 to 40. 
DOI: $10.17148 /$ IARJSET.2021.8841

Now, our aim is to train the data set using Machine Learning. Machine Learning accounts to those computer algorithms that improve through experience [9]. It can be visualized as a subset of Artificial Intelligence. Machine learning algorithms are known to build a mathematical model based on sample data that are famously known as "training set" which allows them to make decisions without being explicitly programmed to do so upon a target data called as "test set"[9]. A plethora of machine learning algorithms were presented in [5-6] and they are Linear Regression, Logistic Regression, Decision Tree, SVM, Naive Bayes, k-nearest neighbors, K-Means, Random Forest, Dimensionality Reduction Algorithms, neural network and Gradient Boosting algorithms.

Among them, a study made in [9] confirmed that Neural Networks predicted with $99.9 \%$ precision in a similar ranking scenario. This motivated us to deploy the same AI approach in our work. Once the training is accomplished, the mean values for all 17 ranking parameters are calculated and is furnished into a mean quintuple set $\left(\mathrm{Q}_{\mathrm{m}}\right)$.

$$
Q_{m}=\left(t_{m}, r_{m}, g_{m}, o_{m}, p_{m}\right)
$$

Next, a target institution is chosen. The target institution is your HEI, whose parameter is to be bettered. For our study, we chose the one at the bottommost position ( $40^{\text {th }}$ rank) in the NIRF 2020 ranking of Medical colleges. Each value of the $\mathrm{Q}_{40}$, i.e.., $\mathrm{t}_{40}, \mathrm{r}_{40}, \mathrm{~g}_{40}, \mathrm{O}_{40}$ and $\mathrm{p}_{40}$ are compared to the corresponding values in mean quintuple set $\left(\mathrm{Q}_{\mathrm{m}}\right)$. The comparison is not a straight-forward task as each value of the quintuple set has sub-parameters. For this, we employ the deep learning Neural Network algorithm to find if the value of a HEIs quintuple is greater than the mean quintuple set $\left(Q_{m}\right)$. Then we replace each of the 17 parametric values of the target institution with mean quintuple set $\left(Q_{m}\right)$ in 5 steps (one for each parameter). After each step, we compute modified rank of the HEI. This again results us with another quintuple. This is modified rank quintuple (QR). The original NIRF rank of the HEI is compared to each value of modified rank quintuple $(\mathrm{QR})$ set. The better rank among them is noted and the corresponding parameter is the top concern (TP) for that particular institution. The algorithm is given in TABLE 1.

Table 1. Our work

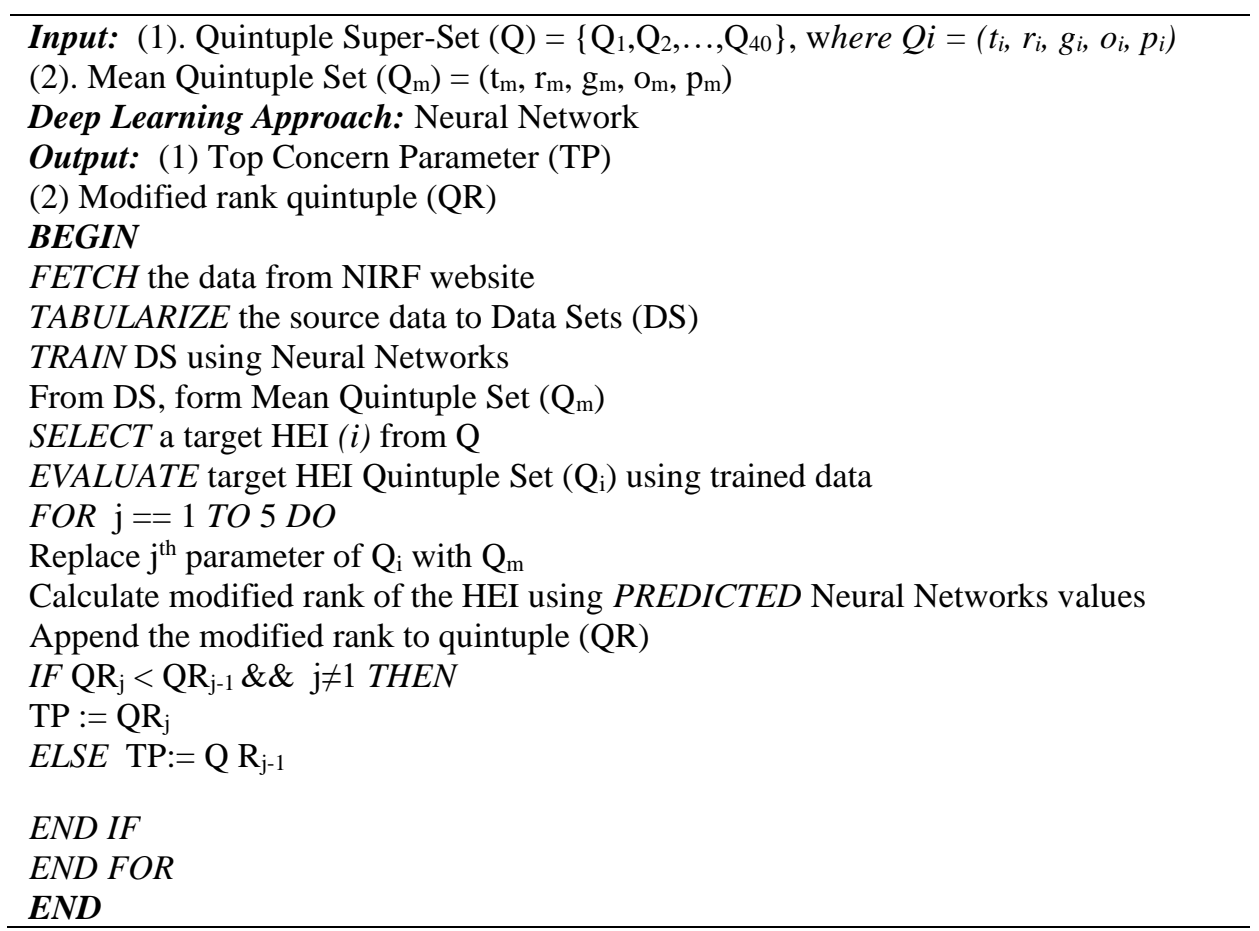

\section{RESULTS AND ANALYSIS}

In this section, we attempt to debrief the simulation conducted for our work. We conducted the simulation in Orange data science toolkit $[9,10]$. From the official website of NIRF, we fetched the data containing the individual weighted scores of parameters. As mentioned in Section 1 of the paper, we have mentioned that there are 9 categories in total for NIRF. Among them we have chosen Medical ranking category. Also, we chose the most recent ranking in June 2020. 


\section{International Advanced Research Journal in Science, Engineering and Technology}

Vol. 8, Issue 8, August 2021

DOI: 10.17148/IARJSET.2021.8841

From the fig. 2, it is evident that we chose 5 major AI approaches to find the optimal one among them. This simulation rendered us with the confusion matrices of all these 5 deep learning approaches.

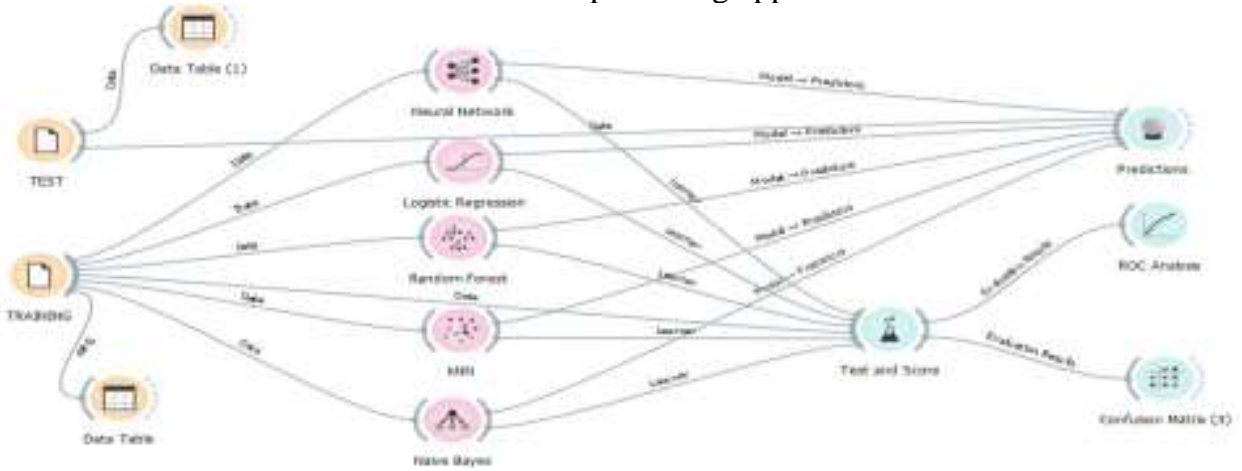

Fig.2. Orange data Science toolkit - Canvas of widgets

The values of fig 3, employing different scoring frameworks which includes CA (Accuracy classification score), Precision (to compute precision), recall (to compute true positives and false negatives), F1 score (weighted average of the precision and recall) and AUC (stored predictions and actual data in model testing).

The NIRF 2020 for medical HEIs is topped by All India Institute of Medical Sciences (AIIMS) among 39 other medical institutes. For the sake of experimentation purposes, we chose 10 sample ranks as the target HEIs. We took up two broader case studies for our simulation. First group ranks were 40, 39, 38, 37and 36. But since there was two HEIs in 38th position, there is not 39th rank. Second group ranks we chose were 35, 30, 25 and 20.

The last ranked HEI of the medical category in 2020 is our first target college. As per our algorithm, we took the parameters (all 5 major parameters with 17 sub-parameters) from the website and tabularized them into manageable data sets, using which serves as input to our work. With this dataset, we formed the Quintuple set of the institute. Next, we replaced the first parameter TLR with the corresponding TLR parameter in the Mean Quintuple set. After replacement, the rank of the last ranked NIRF institution, still unaltered, is at 40th position. The same process is repeated for other parameters as well. It was found that the ranks stayed the same for parameters RP, OI and PR.

$\begin{array}{llrlrrr}\text { Model } & \text { AUC } & \text { CA } & \text { F1 } & \text { Precision } & \text { Recall } \\ \text { kNN } & 0.9437660668380464 & 0.15 & 0.071666666666666667 & 0.04875 & 0.15 \\ \text { Random Forest } & 0.9993573264781495 & 0.925 & 0.8983333333333334 & 0.8833333333333332 & 0.925 \\ \text { Neural Network } & 1.0000000000000004 & 1.0 & 1.0 & 1.0 & 1.0 \\ \text { Naive Bayes } & 1.0000000000000004 & 0.975 & 0.975 & 0.9875 & 0.975 \\ \text { Logistic Regression } & 1.0000000000000004 & 1.0 & 1.0 & 1.0 & 1.0\end{array}$

Fig.3. Orange data Science toolkit - Evaluation Results

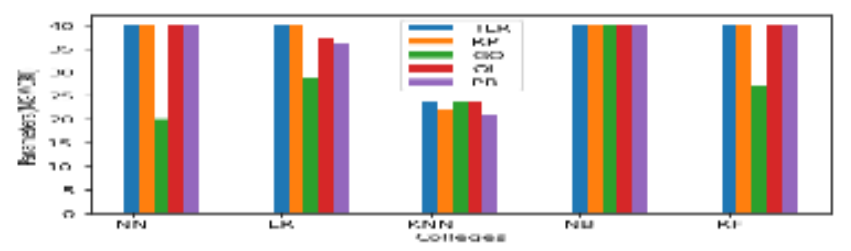

Fig.4. NIRF ranking parameters in all 5 deep learning approaches

For the HEI with the rank 37, there is a concern to upgrade their GO. With the mean value of GO, the college could move up to 29th rank. For the HEI with the rank 36, there is a concern to upgrade their RP aiding which their college could move up to 31 st rank. The graphical results implemented in python framework [8, 9 and 10] are shown in fig [5,6]. For the second group of HEIs belonging to ranks 35, 30, 25 and 20, there was not significant improvement of ranks with respect to altering any parametric score except for rank 30 for a single parameter. The resulting graph is displayed in fig 6 . 
DOI: $10.17148 /$ IARJSET.2021.8841

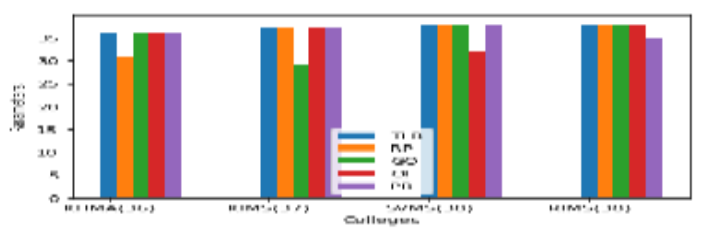

Fig.5. NIRF for last 5 ranked HEIs with respect to ranking parameters

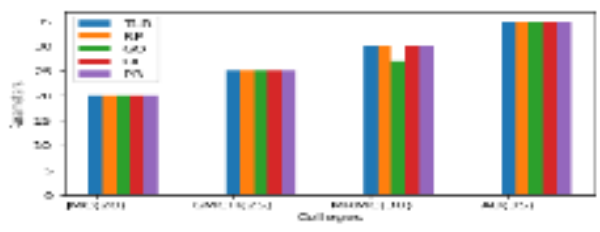

Fig.6. NIRF for the second group of HEIs with respect to ranking parameters

\section{$5 \quad$ AN OPEN DISCUSSION AND FUTURE WORK}

In this paper, we have successfully implemented a deep learnt business model that can be employed by HEIs across the country for the betterment of their NIRF scores. Without the shadow of ambiguity, the admission-seekers are well informed these days through the internet and social media and they check the grade of a college before joining an institute. So, it is at the top priority that the college must earn better scores in the forthcoming year. The reason behind naming it a business model is because a college management can allocate appropriate financial share to upgrade the facilities and/or human/material resources pertaining to that ranking parameter that is down-under.

\section{REFERENCES}

1.A. Kumar and S. K. Tiwari, "India rankings 2016: Ranking model for Indian higher educational institutions," 2016 International Conference on ICT in Business Industry \& Government (ICTBIG), Indore, 2016, pp. 1-6, doi: 10.1109/ICTBIG.2016.7892716.

2.A. M. and M. Nagabhushanam, "A Ranking Framework for Higher Education Institutions in India: A Policy Analysis," $20175^{\text {th }}$ IEEE International Conference on MOOCs, Innovation and Technology in Education (MITE), Bangalore, 2017, pp. 27-30, doi: 10.1109/MITE.2017.00010.

3.B. Sudakova, M. Nazaruk, N. Kunanets, A. Rzheuskyi, V. Pasichnyk and Y. Bilak, "The Project of Intellectual System for Rating of Educational Institutions," 2019 IEEE 14 ${ }^{\text {th }}$ International Conference on Computer Sciences and Information Technologies (CSIT), Lviv, Ukraine, 2019, pp. 63-66, doi: 10.1109/STC-CSIT.2019.8929884.

4.T. Suksida and L. Santiworarak, "A study of website content in webometrics ranking of world university by using similar web tool," 2017 IEEE $2^{\text {nd }}$ International Conference on Signal and Image Processing (ICSIP), Singapore, 2017, pp. 480-483, doi: 10.1109/SIPROCESS.2017.8124588.

5.K. L. Chugh, N. Alekhya, N. P. Kishore and K. Shekar, "Analysis of National Institutional Ranking Framework-Journey so far," 2018 IEEE $6^{\text {th }}$ International Conference on MOOCs, Innovation and Technology in Education (MITE), Hyderabad, India, 2018, pp. 115-119, doi: 10.1109/MITE.2018.8747105.

6.Anisha Kumari , https://www.ndtv.com/education/nirf-ranking-2020-live-update, June 11, 2020.

7.MHRD India, https://www.nirfindia.org/Docs/Ranking-Framework-for-Engineering-Institutions.pdf, DOA: $25^{\text {th }}$ February 2021.

8.Tom Mitchell, "Machine Learning", Tata McGraw Hill, ISBN 0070428077, 1997.

9.Terrance Frederick Fernandez and M. Pradeep, "Multi-level Predictive with Training Framework (MP with TF) for ranking machine learning algorithms", IEEE proceeding of $4^{\text {th }}$ International conference on I-SMAC (IoT in Social, Mobile, Analytics and Cloud (I-SMAC 2020), pp.697-703, ISBN: 978-1-7281-5464-0/20, $7^{\text {th }}-9^{\text {th }}$ Oct 2020, SCAD Palladum, Tamil Nadu.

10.Yamamoto, Hiroaki, Youji Kanie, Masakazu Arakawa, and Keiji Taniguchi. "Theoretical study of resonant tunneling in rectangular double-, triple-, quadruple-, and quintuple-barrier structures." Applied Physics A 50, no. 6 (1990): 577-581.

11.Amit Kumar Tyagi, Aswathy S U, Terrance Frederick Fernandez, N. Sreenath and Shashvi Mishra "Prediction of Chemicals and fertilizers using machine learning and IoT", IEEE proceeding of $11^{\text {th }}$ International Conference on Computer Science and Informatics, 27th - 29th January 2021, Coimbatore, Tamil Nadu

12.Bhavya Gudeti, Terrance Frederick Fernandez, Shasvi Mishra, Amit Kumar Tyagi, Shaveta Malik and Shabnam Kumari, "A Novel Approach to Predict Chronic Kidney Disease using Machine Learning Algorithms", IEEE proceeding of $4^{\text {th }}$ International Conference on Electronics, Communication and Aerospace Technology (ICECA 2020), ISBN: 978-1-7281-6387-1, Coimbatore, Tamil Nadu.

13.Amit Kumar Tyagi, Shabnam Kumari, Terrance Frederick Fernandez and Aravindan .C, "P3 Block: Privacy Preserved, Trusted Smart Parking Allotment for Future Vehicles of Tomorrow", Springer proceeding of International Conference on Computational Science and Its Applications, Part VI, Chapter 56, LNCS 12254, pp.783-796, ISBN: 978-3-030-58816-8, https://doi.org/10.1007/978-3-030-58817-5_56, 1st - 4th July 2020, Università degli Studi di Cagliari, CA, ITALY.

14.Aravindan C, Terrance Frederick Fernandez, Hema Malini V and Catherine Madhu Vidha J, "An Extensive Research on Cyber Threats using Learning Algorithm", IEEE proceeding of International Conference on Emerging Trends in Information Technology and Engineering, ISBN: 978-17281-4141-1, 25th February 2020, VIT, Vellore, India. 


\section{International Advanced Research Journal in Science, Engineering and Technology}

Vol. 8, Issue 8, August 2021

DOI: $10.17148 /$ IARJSET.2021.8841

15.Yamamoto, Hiroaki, Youji Kanie, Masakazu Arakawa, and Keiji Taniguchi. "Theoretical study of resonant tunneling in rectangular double-, triple-, quadruple-, and quintuple-barrier structures." Applied Physics A 50, no. 6 (1990): 577-581.

16.Geerthana R.; Nandhini P.; Suriyakala R.. "Medicinal Plant Identification Using Deep Learning". International Research Journal on Advanced Science Hub, 3, Special Issue ICITCA-2021 5S, 2021, 48-53.

17.Krithika G K; Karthik S; Kowsalya R; Alfred Daniel J; Sangeetha K. "Driver Alert System Using Deep Learning and Machine Learning". International Research Journal on Advanced Science Hub, 3, Special Issue ICARD-2021 3S, 2021, 120-123. doi: 10.47392/irjash.2021.078

18.Sona Solanki; Asha D Solanki. "Review of Deployment of Machine Learning in Blockchain Methodology". International Research Journal on Advanced Science Hub, 2, 9, 2020, 14-20. doi: 10.47392/irjash.2020.141 